\title{
Three-Dimensional Characterization of Magnetic Tunnel Junctions for Read-Head Applications by Atom-Probe Tomography
}

\author{
D.K. Schreiber, ${ }^{* * *}$, Y-S. Choi, ${ }^{* * *}$ Y. Liu, ${ }^{* *}$ D.N. Seidman,, ${ }^{* * * *}$ and A.K. Petford-Long ${ }^{*, * *}$ \\ * Dept. of Mat. Sci. \& Engin., Northwestern University, 2220 Campus Dr., Evanston, IL 60208 \\ ** Materials Science Division, Argonne National Laboratory, 9700 S. Cass Ave, Argonne, IL 60439 \\ *** Electronic Devices Engineering HQ, Canon ANELVA Corporation, Kawasaki 215-8550 Japan \\ ${ }^{* * * *}$ Northwestern University Center for Atom-Probe Tomography (NUCAPT), Evanston, IL 60208
}

Complex, thin-film heterostructures play a critical role in a wide range of modern microelectronic devices. The continual reduction of the characteristic dimensions of these systems has, however, made it increasingly difficult and yet vitally essential to both characterize and understand the role of microstructure and, in particular, of layer interfaces on the resulting electrical, optical, and magnetic properties of these systems. One such materials system of interest is the magnetic tunnel junction (MTJ) used as the read-head sensor in hard disk drives and also as the memory element in magnetic random access memories [1]. While significant research efforts have been made toward understanding this materials system [2] many questions remain due to the complexity and nanometer scale dimensions of the system. In this research, atom-probe tomography (APT) is utilized to investigate the three-dimensional nanostructure of MgO-based MTJs.

The samples were CoFeB-MgO MTJs that included all the key layers and thicknesses used in practical devices. The stack comprised: $\mathrm{Si} / / \mathrm{Seed} / \mathrm{PtMn}(20) / \mathrm{Co}_{70} \mathrm{Fe}_{30}(2.5) / \mathrm{Ru}(0.9) /$ $\mathrm{Co}_{60} \mathrm{Fe}_{20} \mathrm{~B}_{20}(3) / \mathrm{MgO}(1.1) / \mathrm{Co}_{60} \mathrm{Fe}_{20} \mathrm{~B}_{20}(8) / \mathrm{Ru}(1) / \mathrm{Co}(15) / \mathrm{Fe}(55)$ (thickness in nm). The magnetotransport properties of this stack were optimized for read-head applications. Samples were analyzed in both the as-grown state and after annealing at $380^{\circ} \mathrm{C}$ for $2 \mathrm{hrs}$. Prior to annealing, B was uniformly distributed laterally within the $\mathrm{CoFeB}$ layers and exhibited some segregation toward the $\mathrm{CoFeB}-\mathrm{on}-\mathrm{Ru}$ and $\mathrm{CoFeB}-\mathrm{on}-\mathrm{MgO}$ interfaces, Fig 1. Annealing resulted in partial crystallization of the $\mathrm{CoFeB}$ and diffusion and segregation of $\mathrm{B}$ within the $\mathrm{CoFeB}$ layers into a three-dimensional grain structure, Fig 2. The formation of both crystalline and amorphous grains upon annealing has been seen previously with TEM [3], but for the first time with APT we have been able to measure directly the composition of each individual grain. The amorphous grain contained $\sim 12$ at. $\% \mathrm{~B}$, while the crystalline grain contained $\sim 3$ at. $\%$ B. The crystallinity of the B-poor grain was confirmed by the presence of atomic planes within the APT reconstruction of that region. Unexpectedly, the $\mathrm{Co}:(\mathrm{Co}+\mathrm{Fe})$ ratio within each grain was also quite different, Fig 3 . The crystalline grain exhibited a significantly higher Fe concentration than the amorphous grain, which would affect the resulting tunneling spin polarization of an MTJ device. Intriguingly, APT analysis of a similar MTJ that excluded the $1 \mathrm{~nm}$ Ru cap, adjacent to the top CoFeB layer, displayed no lateral segregation of B within the $\mathrm{CoFeB}$, highlighting the critical importance of the thin $\mathrm{Ru}$ capping layer on the resulting three-dimensional nanostructure and device performance.

[1] J. G. J. Zhu and C. D. Park, Materials Today 9, 36-45 (2006).

[2] J. J. Cha et al., Applied Physics Letters 95, 032506 (2009).

[3] T. Miyajima et al., Applied Physics Letters 94, 122501 (2009).

[4] This work was supported under Contract No. DE-AC02-06CH11357 1357 between Chicago Argonne, LLC and the Department of Energy. The atom-probe tomography was performed at the Northwestern University Center for Atom-Probe Tomography (NUCAPT) and the electron microscopy was performed at the Electron Microscopy Center at Argonne National Laboratory. 

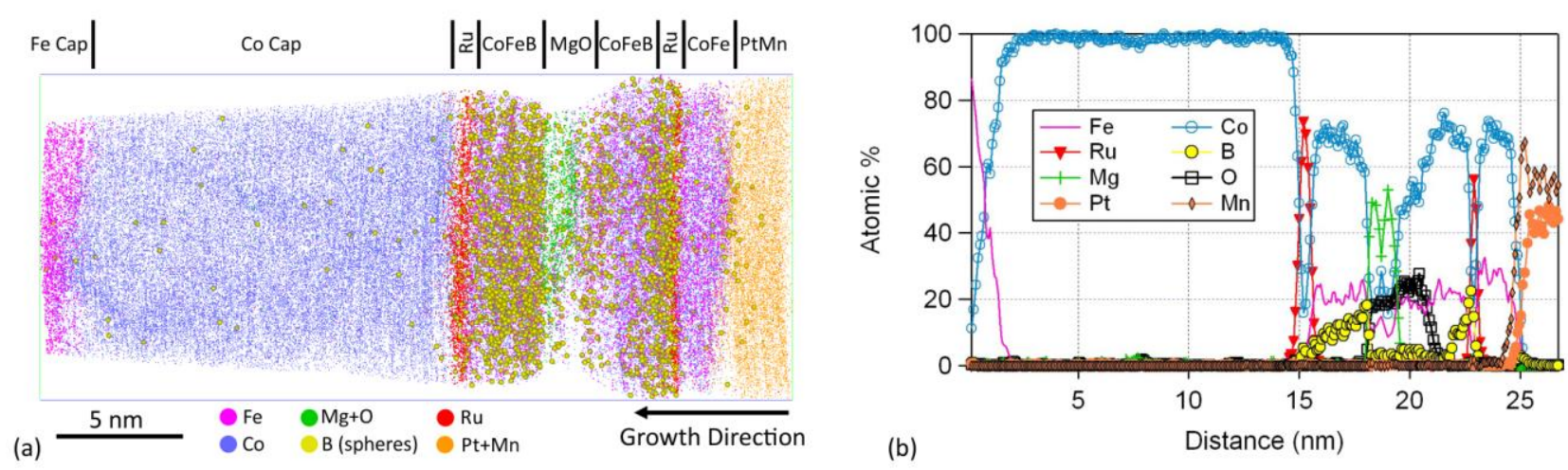

FIG 1: (a) APT reconstruction of an as-grown MTJ structure. (b) 1D concentration profile of the same dataset. $\mathrm{B}$ is uniformly distributed laterally within each $\mathrm{CoFeB}$ layer, but has segregated preferentially at the $\mathrm{CoFeB}-\mathrm{on}-\mathrm{Ru}$ and $\mathrm{CoFeB}-o n-\mathrm{MgO}$ interfaces. The oxygen tail extending below the $\mathrm{MgO}$ has been shown, at least in part, to be an artifact of the experiment and will be discussed.
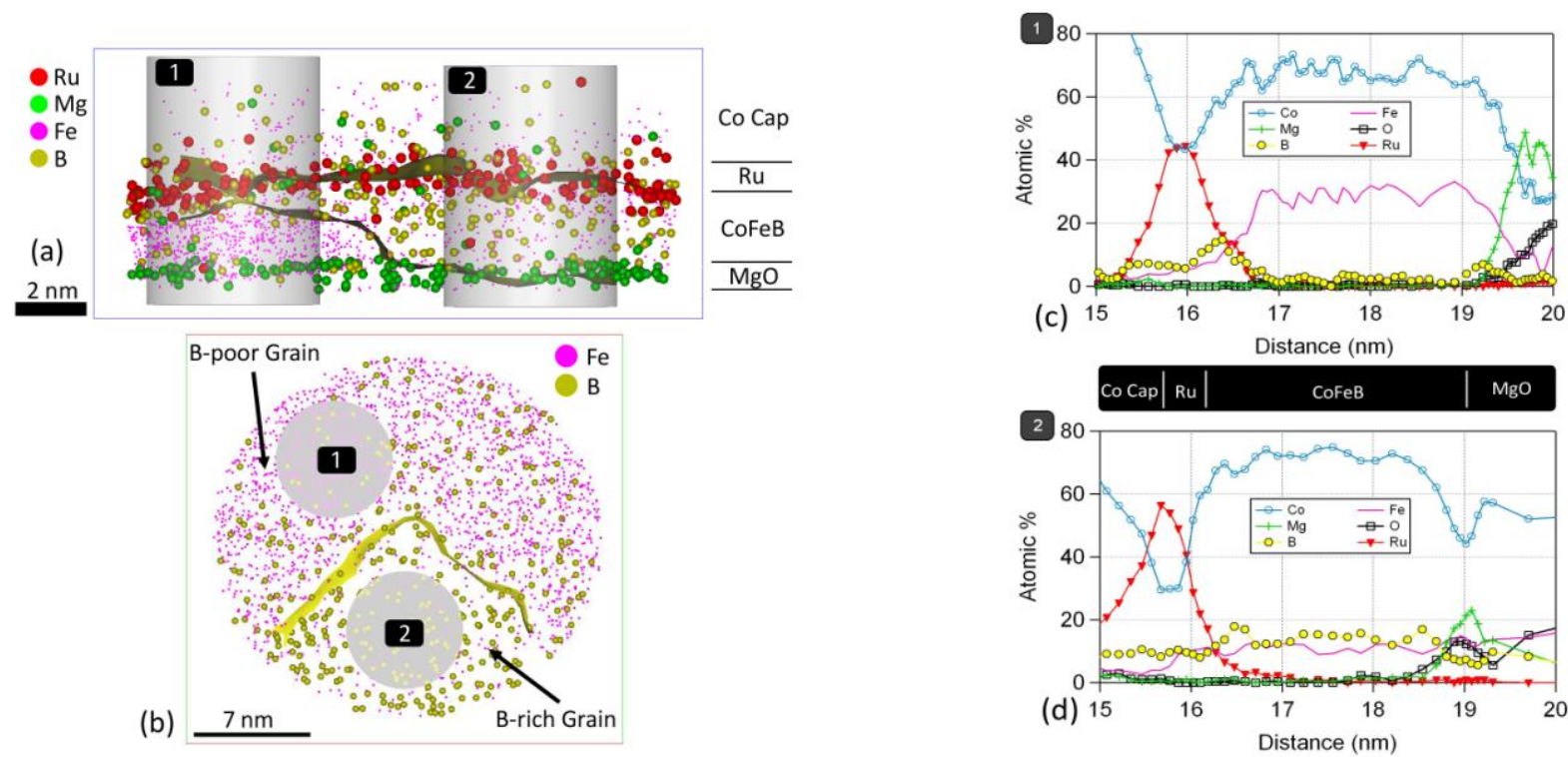

FIG 2: (a) A $1 \mathrm{~nm}$ thick cross-section and (b) plan-view slice of the upper CoFeB layer after annealing. An 8 at.\% B isoconcentration surface clearly delineates a grain boundary between a (1) crystalline and (2) an amorphous $\mathrm{CoFeB}$ grain. 1D concentration profiles, (c) and (d), reveal significant compositional differences in $\mathrm{B}$ and Fe concentrations between the two grains.

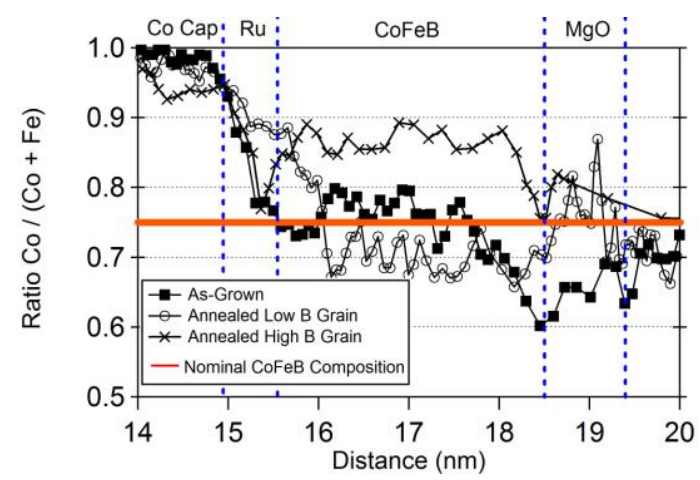

FIG 3: A 1D profile of the $\mathrm{Co}:(\mathrm{Co}+\mathrm{Fe})$ ratio shows further composition differences between the two grains. This composition variance will affect the spin polarization of the tunneling current and thus the magnetoresistance of the device. 